\title{
Identification of conserved, centrosome-targeting ASH domains in TRAPPII complex subunits and TRAPPC8
}

\author{
Kenneth B Schou ${ }^{1,2}$, Stine K Morthorst ${ }^{1}$, Søren T Christensen ${ }^{1}$ and Lotte B Pedersen ${ }^{1 *}$
}

\begin{abstract}
Background: Assembly of primary cilia relies on vesicular trafficking towards the cilium base and intraflagellar transport (IFT) between the base and distal tip of the cilium. Recent studies have identified several key regulators of these processes, including Rab GTPases such as Rab8 and Rab11, the Rab8 guanine nucleotide exchange factor Rabin8, and the transport protein particle (TRAPP) components TRAPPC3, -C9, and -C10, which physically interact with each other and function together with Bardet Biedl syndrome (BBS) proteins in ciliary membrane biogenesis. However, despite recent advances, the exact molecular mechanisms by which these proteins interact and target to the basal body to promote ciliogenesis are not fully understood.

Results: We surveyed the human proteome for novel ASPM, SPD-2, Hydin (ASH) domain-containing proteins. We identified the TRAPP complex subunits TRAPPC8, -9, -10,-11, and -13 as novel ASH domain-containing proteins. In addition to a C-terminal ASH domain region, we predict that the N-terminus of TRAPPC $8,-9,-10$, and -11, as well as their yeast counterparts, consists of an a-solenoid bearing stretches of multiple tetratricopeptide (TPR) repeats. Immunofluorescence microscopy analysis of cultured mammalian cells revealed that exogenously expressed ASH domains, as well as endogenous TRAPPC8, localize to the centrosome/basal body. Further, depletion of TRAPPC8 impaired ciliogenesis and GFP-Rabin8 centrosome targeting.
\end{abstract}

Conclusions: Our results suggest that ASH domains confer targeting to the centrosome and cilia, and that TRAPPC8 has cilia-related functions. Further, we propose that the yeast TRAPPII complex and its mammalian counterpart are evolutionarily related to the bacterial periplasmic trafficking chaperone PapD of the usher pili assembly machinery.

Keywords: TRAPPII complex, ASH domain, Vesicle trafficking, Cilia, Rab8, TPR repeat, Golgi, Centrosome, Rabin8, MSP domain

\section{Background}

The primary cilium is a microtubule-based sensory organelle that extends from the mother centriole, projecting from the cell surface into the extracellular environment [1]. The assembly of primary cilia is a multistep process initiated by docking of the centriole distal end to a vesicle or membrane patch that subsequently expands and surrounds the axoneme as it elongates by intraflagellar transport (IFT) $[2,3]$. In many cell types the proximal part of the mature cilium resides in the cytoplasm within an invagination of the plasma membrane known as the ciliary pocket. This region is a unique site for vesicular trafficking

\footnotetext{
* Correspondence: Ibpedersen@bio.ku.dk

${ }^{1}$ Department of Biology, University of Copenhagen, Universitetsparken 13, Copenhagen, Denmark

Full list of author information is available at the end of the article
}

by endo- and exocytosis that plays a critical role in ciliary membrane homeostasis and function $[4,5]$.

Primary cilia have become the focus of mounting studies owing to their role in coordinating cellular signaling processes during development and tissue homeostasis, and consequently, their association with a constellation of genetic diseases and syndromes called ciliopathies $[6,7]$. These include the Bardet-Biedl syndrome (BBS), characterized by obesity, renal anomalies, cognitive defects, post-axial polydactyly, hypogonadism, retinal degeneration, and anosmia [8]. Seven BBS gene products (BBS1, BBS2, BBS4, BBS5, BBS7, BBS8, and BBS9) and BBIP10 reside in a stable complex called the BBSome $[9,10]$. The BBSome interacts with the Rab8 guanine nucleotide exchange factor Rabin8, as well as several other proteins, and mediates trafficking of membrane proteins

\section{Biomed Central}


to and from cilia $[9,11,12]$. Although numerous proteins have been implicated in vesicular transport to cilia (reviewed in [13]), Rab8 seems to be a key player in the very first stages of ciliary membrane biogenesis. Rab8 is targeted to the primary cilium during early ciliogenesis followed by a gradual loss from the cilium as the organelle matures [14]. Rab8 is activated by Rabin8 [15], which is recruited to the centrosome and activated by a mechanism involving Rab11 and homologs of the yeast transport protein particle II (TRAPPII) complex subunits $[14,16]$.

Current evidence indicates that the yeast TRAPPII complex consists of a core containing the seven TRAPPI subunits (Trs20, Trs23, Trs31, Trs33, Bet3a, Bet3b, Bet5) as well as the four TRAPPII-specific subunits Trs65, Trs120, Trs130, and Tca17 $[17,18]$. Functional and biochemical studies have indicated that yeast TRAPPI and TRAPPII complexes are distinct vesicle tethering entities that function in ER-Golgi and intra-Golgi plus endosome-Golgi transport, respectively [17]. Yeast also contains a third TRAPP complex, TRAPPIII, which consists of TRAPPI and Trs 85 and functions in autophagy [17]. The composition and function of mammalian TRAPP complexes is less clear, and there is some uncertainty as to how many distinct TRAPP complexes exist in mammalian cells [17]. It is clear, however, that mammalian homologs of the yeast TRAPPI subunits exist in one or more large complexes that also include several homologs of yeast TRAPPII-specific subunits, including Trs120 (TRAPPC9), Trs130 (TRAPPC10), Tca17 (TRAPPC2L) in addition to the Trs130-related TRAPPC11 (C4orf41) and the Trs65-related TRAPPC13 (C5orf44) [17-19]. Furthermore, although Trs85 seems to be absent from the yeast TRAPPII complex, an ortholog of Trs85 (TRAPPC8) appears to be part of the mammalian TRAPP complex since TRAPPC8 was reported to interact with TRAPPC2 (Trs20 homolog) [20] as well as with TRAPPC2L (Tca17 homolog) and TRAPPC13 [18]. Thus in mammalian cells, TRAPPC8 engages in a complex with both TRAPPI and TRAPPII components. Curiously, TRAPPC8 does not appear to interact with the TRAPPII component TRAPPC9 [14,20], suggesting the existence of distinct TRAPPII-like complexes in mammalian cells. Mammalian TRAPPC8 differs from yeast Trs85 in that it contains a C-terminal extension related to the C-terminus of TRAPPC9, which may explain its association with TRAPPII-specific subunits [18].

Similar to yeast TRAPP complexes, mammalian TRAPP has been implicated in various vesicle trafficking pathways [17]. For example, it was shown that depletion of TRAPPC3, TRAPPC9, or TRAPPC10 in cultured cells impaired primary ciliary membrane biogenesis by inhibiting Rabin8 recruitment to the centrosome [14]. While the possible roles of other TRAPP components in ciliogenesis are unclear, several additional proteins involved in ciliary membrane biogenesis interact with Rab8/Rabin8 (reviewed in [13]). These include the centriole distal appendage protein Cep164 [21] and components of the exocyst complex [22] as well as oculocerebrorenal syndrome of Lowe protein, OCRL1, mutations in which cause a rare X-linked disorder characterized by mental retardation, renal tubulopathy, and congenital cataracts [23]. OCRL1 resides in complexes with various Rab proteins $[24,25]$, with preference for Rab8a, via a binding interface consisting of one $\alpha$-helix and an adjacent ASH (ASPM, SPD-2, Hydin) domain [26], a novel family of remote homologs of the immunoglobulin (Ig)-like seven-stranded beta sandwich fold superfamily of the nematode major sperm proteins (MSPs) $[27,28]$. Although poorly defined at present, the MSP fold is believed to form a proteinprotein interaction interface involved in cellular signaling and trafficking activities [28,29]. A previous computational survey identified 13 human ASH-containing proteins [27], all of which are confined to three subcellular compartments, namely the centrosome, Golgi, and the cilium, suggesting that the ASH domain is involved in cilia-related functions [27]. Indeed, OCRL1 was recently shown to be required for ciliogenesis and to promote protein trafficking to the primary cilium via a Rab8 and IPIP27/ Ses-dependent mechanism [30]. Another ASH-domaincontaining protein, the hydrocephalus-associated protein, Hydin, is required for formation of central pair projections of motile cilia and, in turn, for regulating ciliary motility [31-33], whereas the ASH domain-containing ASPM protein is a centrosomal and microtubule-associated protein that causes microcephaly when defective or absent [34].

We here revisit the ciliary family of ASH domaincontaining proteins in humans and yeast and unveil the existence of ASH domains in several members of the TRAPP complex family of proteins. Our database searches identify conserved ASH domains in the C-terminus of mammalian TRAPPC8, $-9,-10,-11$, and -13 , as well as in the yeast TRAPPII complex proteins Trs65, Trs120, and Trs130, none of which were previously assigned any protein structure. We find that the ASH domain in most of these proteins is preceeded by an $\mathrm{N}$-terminal region containing long $\alpha$-helical matrices exhibiting high levels of helicity and TPR repeat propensity. We further provide evidence that the ASH domains of TRAPPC10 and TRAPPC11 localize to the centrosome when expressed as fusion proteins in mammalian cells and that their expression leads to defects in microtubule organization. Finally, we find that endogenous TRAPPC8 localizes to the centrosome/basal body in cultured cells, and that TRAPPC8 depletion impairs ciliogenesis and targeting of GFP-Rabin8 to the centrosome. Our results corroborate the notion that the ASH domain is involved in microtubule- and ciliarelated processes and provide insight into the mechanism 
by which mammalian TRAPPII homologs target to the centrosome/basal body. Our results further suggest that the yeast TRAPPII complex and its mammalian counterpart are evolutionarily related to the bacterial periplasmic trafficking chaperone PapD of the usher pili assembly machinery [35].

\section{Methods}

\section{Bioinformatics}

Profile-to-profile hidden Markov model (HMM)-HMM searches against the PFAMA database (http://pfam. sanger.ac.uk) were performed using HHpred [36,37] with default settings. Multiple sequence alignments (MSA) were generated using MAFFT $[38,39]$, edited in Jalview [40], and the consensus of the alignment calculated and colored using ClustalX, as implemented in Jalview. Secondary structure information and structural alignment were predicted using HHpred [37]. For homology modeling of three-dimensional (3D) structures, Modeller [41,42] was employed and templates were chosen based on highest probability and significantly low E value. Discovery Studio 3.5 Visualizer was used for analysis of resulting 3D model coordinates.

\section{PCR and cloning procedures}

For generation of plasmids coding for Myc-tagged ASH domains from human TRAPPC10 (amino acid residues 1000-1259) and TRAPPC11 (residues 701-1133; transcript variant 1 ), the corresponding $\mathrm{CDNA}$ regions were PCRamplified from retinal pigment epithelial cell cDNA [43] by standard procedures using forward (CAGAATTC TCCCCATCTACAGCAAGCAGTC for TRAPPC10; CA GAATTCTCTTAAATTGGCAGGGAGGAGGAGGA for TRAPPC11) and reverse (CAGGTACCTCATGTTACAC TGACTTCCAGG for TRAPPC10; CAGGTACCTCATG CAGCAGCAATAGAGGTAT for TRAPPC11) primers containing EcoR1 and Kpn 1 restriction sites (italics), respectively. The PCR products were cloned into pCMVMyc (Clontech laboratories, Inc.) and transformed into Escherichia coli $\mathrm{DH} 10 \alpha$ using standard procedures. Plasmids from recombinant bacteria were purified using endotoxin-free plasmid DNA purification kit (NucleoBond Xtra Midi EF) from Macherey-Nagel and the inserts sequenced at Eurofins MWG Operon.

\section{Mammalian cell culture}

The retinal pigment epithelial (RPE) cells used (lab stock) were derived from the immortalized hTERT RPE-1 cellline and cultured as described previously [43].

\section{Immunofluorescence microscopy}

For immunofluorescence microscopy analysis of cells expressing ASH domain fusion proteins RPE cells were seeded on coverslips, transfected with plasmids encoding
Myc-TRAPPC10-ASH or Myc-TRAPPC11-ASH (see above) and serum starved for $24 \mathrm{~h}$. Cells were fixed with methanol or 4\% PFA and subjected to immunofluorescence microscopy as described [43] using rabbit monoclonal antibody specific for Myc (1:500 dilution; Cell Signaling) and mouse monoclonal antibodies specific for $\alpha$-tubulin (1:4,000 dilution; Sigma), acetylated-tubulin (1:4,000 dilution; Sigma) or $\mathrm{p} 150^{\text {Glued }}$ (1:250 dilution; BD Biosciences). To study the localization of endogenous TRAPPC8 RPE cells were seeded on coverslips and incubated in serumdepleted medium for $24 \mathrm{~h}$ to induce cilia formation. Cells were fixed with methanol and subjected to immunofluorescence microscopy as described [43] using rabbit polyclonal antibody specific for TRAPPC8 (1:100 dilution; Sigma), rat monoclonal antibody specific for EB3 (1:300 dilution; Absea clone KT36), and mouse monoclonal antibodies specific for acetylated-tubulin (1:5,000 dilution; Sigma) and $\mathrm{p} 150^{\text {Glued }}$ (1:500 dilution; BD Biosciences). Imaging was performed with a motorized Olympus BX63 upright microscope equipped with a DP72 color, 12.8 megapixel, $4140 \times 3096$ resolution camera and differential interference contrast (DIC). The software used was Olympus CellSens dimension. Images were processed for publication using Adobe Photoshop CS4 version 11.0.

TRAPPC8 knock-down, GFP-Rabin8 expression, SDS-PAGE, and western blot

For TRAPPC8 knock-down experiments, RPE cells were seeded and subjected to transfection with $100 \mathrm{nM}$ esiRNA specifically targeting TRAPP8C (Cat\# EHU065741; Sigma) or control siRNA (5'-UAA UGU AUU GGA ACG CAU ATT-3'; Eurofins MWG Operon) using DharmaFECT Duo Transfection Reagent (Thermo Scientific) essentially as described [43]. Cells were then incubated in serumdepleted medium for $24 \mathrm{~h}$ and either PFA-fixed and analyzed by immunofluorescence microscopy with acetylated tubulin antibody as described above or lysed using $0.5 \%$ SDS; the lysates were subsequently analyzed by SDS-PAGE and western blotting as described previously [44]. Rabbit polyclonal antibody specific for TRAPPC8 (1:500 dilution; Sigma) and mouse monoclonal antibody specific for $\alpha$ tubulin (1:2,000 dilution; Sigma) were used for western blot. Blots were scanned and processed for publication using Adobe Photoshop CS6 version 13.0 and Adobe Illustrator CS6 version 16.0.0. For experiments with GFP-Rabin8 plasmid RPE cells were seeded and transfected with $100 \mathrm{nM}$ esiRNA or control siRNA as described above. After $30 \mathrm{~h}$, cells were transfected with plasmid encoding GFP-Rabin8 [14] for another $16 \mathrm{~h}$. Prior to fixation with PFA cells were serum starved for $1 \mathrm{~h}$ and subjected to immunofluorescence microscopy with $\mathrm{p} 150^{\text {Glued }}$ antibody as described above, and the number of GFP-positive centrosomes in GFP-Rabin8 expressing cells was scored. 


\section{Results}

Identification of ASH domains in the C-terminus of known and putative TRAPPII components

Sequence homologies between the ASH domain and yeast TRAPPII-specific components and their mammalian counterparts were readily attained by searching the PFAM database of protein families [45] using the human DLEC1 amino acid sequence as a search query in the HHpred server (http://toolkit.tuebingen.mpg.de/hhpred) (Additional file 1: Figure S1). We chose DLEC1 as search query because this protein was identified with high confidence as an ASH domain-containing protein in a bioinformatics analysis [27]. However, the molecular function of DLEC1 is largely unknown. Specifically, a HMM based profile-to-profile search with a minimal portion of human DLEC1 (amino acids 769-980; [27]) carrying the second ASH module produced high probability sequence similarities to the TRAPPC9Trs120 family of the PFAM entry PF08626 (local search algorithm: probability 97.47, $\mathrm{E}=0.035$ ), as well as to a profile of bacterial PapD/FimC (PF14874) and its eukaryotic derivative, the MSP domain (PF00635) (both having $\mathrm{E}<1 \times 10^{-5}$ ) [28]. Since we found that the ASH and MSP domains resemble the same conserved domain family, we have chosen to use the terms ASH and MSP interchangeably. An analogous search using the global search mode recovered essentially the same matches yielding higher score parameters to TRAPPC9-Trs120 (probability 98.10, $\mathrm{E}=3.6 \times 10^{-5}$ ). This indicates that the family of TRAPPII proteins, encompassing metazoan TRAPPC9 homologs and yeast Trs120, bear homology by sequence to the ASH domain. Indeed, a reciprocal profile-to-profile search seeded with, for example, yeast Trs120 (amino acids 660948) identified, besides TRAPPC9-Trs120, the PapD family as the highest scoring hit (local/global search modes: probability 97.78/97.22, $\left.\mathrm{E}=8.6 \times 10^{-4} / 4.4 \times 10^{-4}\right)$. Likewise, using a longer stretch of the Trs120 C-terminus (amino acids 486-1166) as a search query, employing three MSA generation iterations, we identified a portion of human Hydin (amino acids 361-892) containing three ASH domains (global search probability 96.50, $\mathrm{E}=0.017$ ), supporting the existence of the ASH domain in Trs120/TRAPPC9. Interestingly, this search also identified remote sequence similarities to additional or putative TRAPPII subunits, including human TRAPPC13/C5orf44 (local probability 99.41, $\mathrm{E}=9.2 \times 10^{-12}$ ), C4orf44/Gryzun (TRAPPC11) (local probability $99.39, \mathrm{E}=1.1 \times 10^{-9}$ ) as well as TRAPPC8 (local probability $100, E=7.8 \times 10^{-32}$ ). These findings raise the possibility that mammalian TRAPP possesses multiple paralogous ASH domain-bearing components.

Encouraged by the above findings, and to uncover the full repertoire of Trs120/TRAPPC9 homologs bearing ASH domains in humans and yeast, we decided to validate the occurrence of this domain in each of the known or putative TRAPPII-specific subunits. In each instance, the minimal
C-terminal portion displaying sequence homology to ASH was retrieved and used as a query in HHpred (http://toolkit. tuebingen.mpg.de/hhpred). Remarkably, we were able to show the presence of one or two ASH domains in three of the subunits of yeast TRAPPII (Trs65, -120 , and -130) and their human counterparts TRAPPC9, $-10,-11$, and -13 as well as TRAPPC8 (Figure 1). These searches yielded significant local $\mathrm{E}$ values in the range of $10^{-2}-10^{-3}$ with the exception of TRAPPC10 showing more remote similarity to the ASH (probability 91.01 and $\mathrm{E}=0.85$ ). Collectively, these searches show that almost all the known and putative TRAPPII-specific components exhibit genuine homology to the ASH/MSP domain (Figure 1). Further, using the Modeller server (http://toolkit.tuebingen.mpg.de/modeller) we could predict a tertiary structure model of each identified ASH domain that was compatible with the solved 3D structure of the human OCRL1 ASH domain [26] (Figure 2 and data not shown). We also confirmed the human OCRL1 $\mathrm{ASH}$ region as the best, and statistically most significant, match by mining the Protein Data Bank (PDB) [46] for similar 3D structures, thus reinforcing our predictions of the existence of this domain in TRAPPC $8,-9,-10,-11$, and -13 .

\section{Identification of TPR repeats in the $\mathrm{N}$-terminus of known and putative TRAPPII components}

Interestingly, Trs120 based profile-profile searches also revealed significant similarities of its $\mathrm{N}$-terminus to the $\mathrm{N}$-termini of other TRAPPII components as well as to human Rapsyn (probability 97.75, $\mathrm{E}=0.0096$ ) that consist of short tracts of tetratricopeptide (TPR) repeats, indicating that $\operatorname{Trs} 120$ bears TPR repeats in its $\mathrm{N}$-terminus. To further examine the TRAPPII complex subunits for the cooccurrence of TPR repeat conformations, the N-terminal portion of each polypeptide sequence was probed for the presence of high $\alpha$-helical content coinciding with TPR repeat propensity. With the exception of TRAPPC13, all human and yeast TRAPPII subunits were predicted to harbor amino termini containing long $\alpha$-helical matrices exhibiting high levels of helicity $(>50 \%)$ and high TPR repeat propensity, as judged by secondary degrees structure prediction algorithms at the HNN server [47] and searches with the TPRpred server [48]. These results suggest that TRAPPC8, -9, -10, and -11 consist of an $\alpha$-solenoid bearing stretches of multiple TPR repeats followed by one or two ASH domains in the C-terminus (Figure 1).

\section{The ASH domains of TRAPPC10 and TRAPPC11 target to the} centrosome and affect microtubule organization

Since the ASH domain seems to be confined to proteins that localize to the centrosome, Golgi, and the cilium [27], and to validate our bioinformatics results, we analyzed the subcellular localization of two of the identified ASH domains, the ASH domain of TRAPPC10 and the second ASH domain of TRAPPC11 (Figure 1). To this end, 


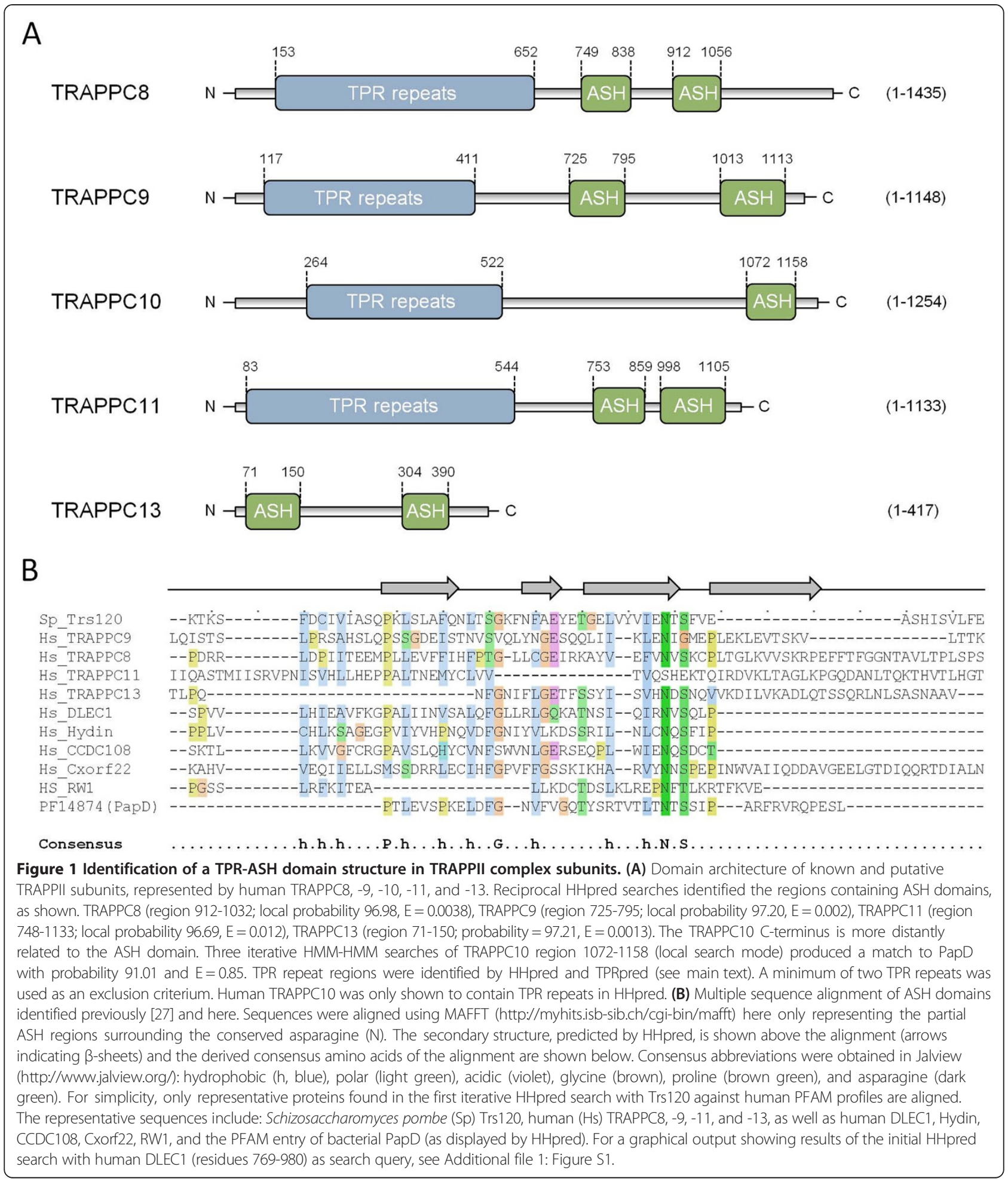

plasmids coding for Myc-tagged versions of these two domains were expressed in RPE cells, the cells subjected to $24 \mathrm{~h}$ of serum starvation to induce ciliogenesis, and analyzed by immunofluorescence microscopy using rabbit antibodies specific for Myc as well as mouse antibodies against $\alpha$-tubulin, acetylated-tubulin, or $\mathrm{p} 150^{\text {Glued }}$ to mark the microtubule cytoskeleton, cilia, and centrosome, respectively. As shown in Figure 3, both Myc-ASH domain fusion proteins localized at the centrosome as well as to specific punctae overlapping the nucleus. Moreover, cells 


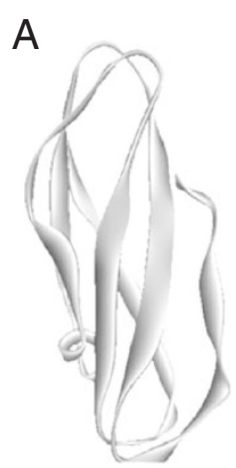

Hydin ASH

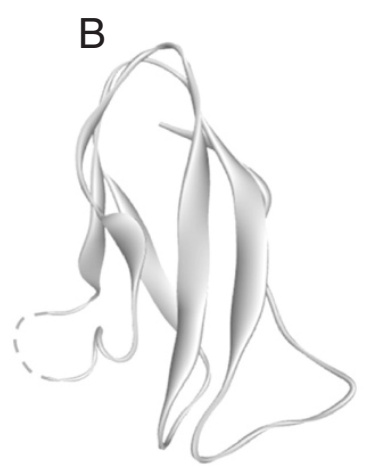

TRAPPC9 ASH

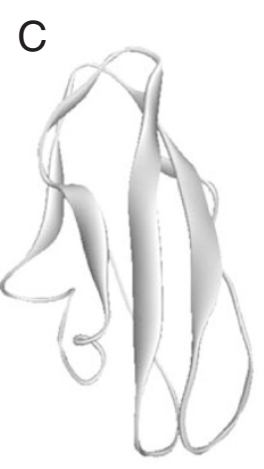

Bacterial PapD

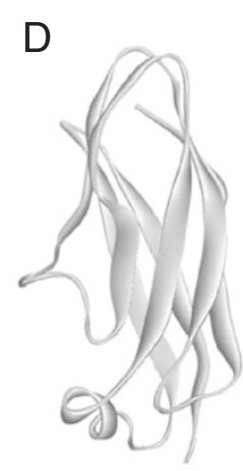

OCRL1 ASH

Figure 2 Predicted 3D structure of the TRAPPII ASH domain. (A) Known structure of a Hydin ASH domain (PDB (http://www.pdb.org) entry 2E6J). (B) Model of the TRAPPC9 ASH structure constructed using Modeller (http://toolkit.tuebingen.mpg.de/modeller) based on the structure of the OCRL1 ASH domain (PDB (http://www.pdb.org) entry 3QBT). (C) The solved structure of the bacterial PapD protein is shown for comparison (PDB (http://www.pdb.org) entry 2WMP). (D) The OCRL1 ASH structural coordinates were obtained at PDB.org (3QBT).

overexpressing the Myc-ASH fusion proteins frequently displayed bundles of microtubules surrounding the nucleus (Figure 3A, B), indicating that overexpression of the ASH domain perturbs microtubule dynamics/organization. We conclude that at least two of the identified ASH domains can target to the centrosome.

\section{Localization of endogenous TRAPPC8 to the centrosome/basal body}

To further investigate the link between the ASH domain and centrosome/basal body localization, we next investigated the subcellular localization of endogenous TRAPPC8 in cultured RPE cells. TRAPPC8 has not previously been shown to localize to centrosomes and cilia; commercially available TRAPPC8 antibody recognized a prominent band of appropriate size in western blot analysis of RPE cell lysate that was reduced in intensity upon treatment of cells with TRAPPC8-specific siRNA (Figure 4A, B). Interestingly, immunofluorescence microscopy analysis of serum-starved RPE cells with the TRAPPC8 antibody showed prominent staining of the centrosome/basal body, as revealed by costaining with antibodies against $150^{\text {Glued }}$, EB3, or acetylated tubulin (Figure 5). Thus TRAPPC8 localizes to the centrosome/basal body. We also used similar approaches to investigate whether TRAPPC11 and TRAPPC13 localize to the basal body/centrosome, but immunofluorescence microscopy analyses with commercially available antibodies against these two proteins were unsuccessful, and hence their subcellular localization could not be addressed. To test if TRAPPC8 is involved in ciliogenesis, as reported previously for TRAPPC3, TRAPPC9, and TRAPPC10 [14], we depleted TRAPPC8 from RPE cells using esiRNA (Figure 4B), subjected cells to serum depletion for $24 \mathrm{~h}$ to induce ciliogenesis, and analyzed ciliation frequency by immunofluorescence microscopy with acetylated tubulin antibody. Interestingly, this analysis revealed a significant reduction in ciliation frequency of TRAPPC8-depleted cells compared to mock-transfected control cells (approximately $33 \%$ and $63 \%$ ciliated cells, respectively; Figure 4C). To investigate if the observed reduction in ciliation frequency of TRAPPC8-depleted cells was linked to defective targeting of Rabin8 to the centrosome, as reported previously for TRAPPC3, TRAPPC9, and TRAPPC10 [14], we expressed GFP-Rabin8 [14] in RPE cells depleted for TRAPPC8 and in mock-transfected control cells. Interestingly, immunofluorescence microscopy of these cells using $150^{\text {Glued }}$ antibody as centrosome marker revealed that TRAPPC8 depleted cells were impaired in their ability to recruit GFPRabin8 to the centrosome (Figure 4D). Thus TRAPPC8 localizes to the centrosome/basal body and appears to be required for ciliogenesis, likely via recruitment of Rabin8 to the centrosome. Further experiments will be required to determine whether TRAPPC8 functions together with or separately from the TRAPPII complex in this process.

\section{Discussion}

Activation of the Rab11-Rabin8-Rab8 axis relies on the highly conserved Golgi TRAPPII subunits TRAPPC9 and TRAPPC10, which associate and co-localize with Rabin8 to ensure its targeting to the centrosome and proper biogenesis of the ciliary membrane [14]. Although poorly defined in mammals, yeast data have revealed a division of labor between the two analogous TRAPPI and TRAPPII complexes [17]. Yeast TRAPPI consists of seven subunits (Trs20, Trs23, Trs31, Trs33, Bet3a, Bet3b, Bet5) and the TRAPPII complex includes four additional proteins, Trs65, Trs120, Trs130, and Tca17, which comprise the putative yeast orthologs of mammalian TRAPPC13, TRAPPC9, TRAPPC10/11, and TRAPPC2L, respectively [17-19]. TRAPPI mediates ER to early Golgi trafficking whereas the TRAPPII complex performs late Golgi vesicle tethering for a diverse group of membrane proteins [17]. Despite a wealth of functional 


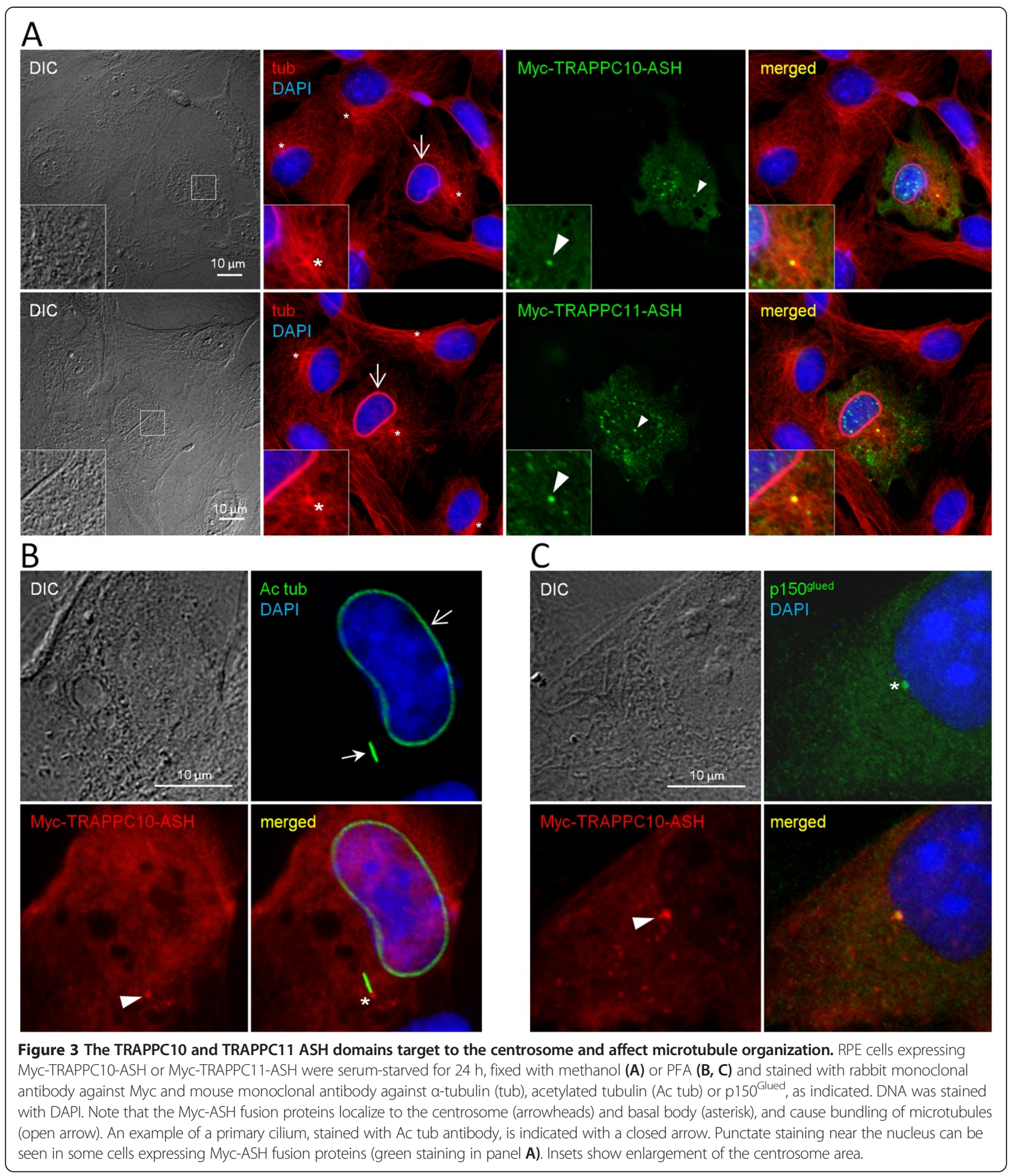

insight, and although the overall architecture of the TRAPPII complex has been determined [49], the molecular structure of individual TRAPPII complex subunits and the biochemical basis for how they recognize their targets remain unknown.
We here shed the first light on the domain organization of conserved TRAPPII complex-specific subunits in yeast their human orthologs with implications for the ciliary targeting and evolution of this protein complex. Based on profile-to-profile searches and structural threading we 


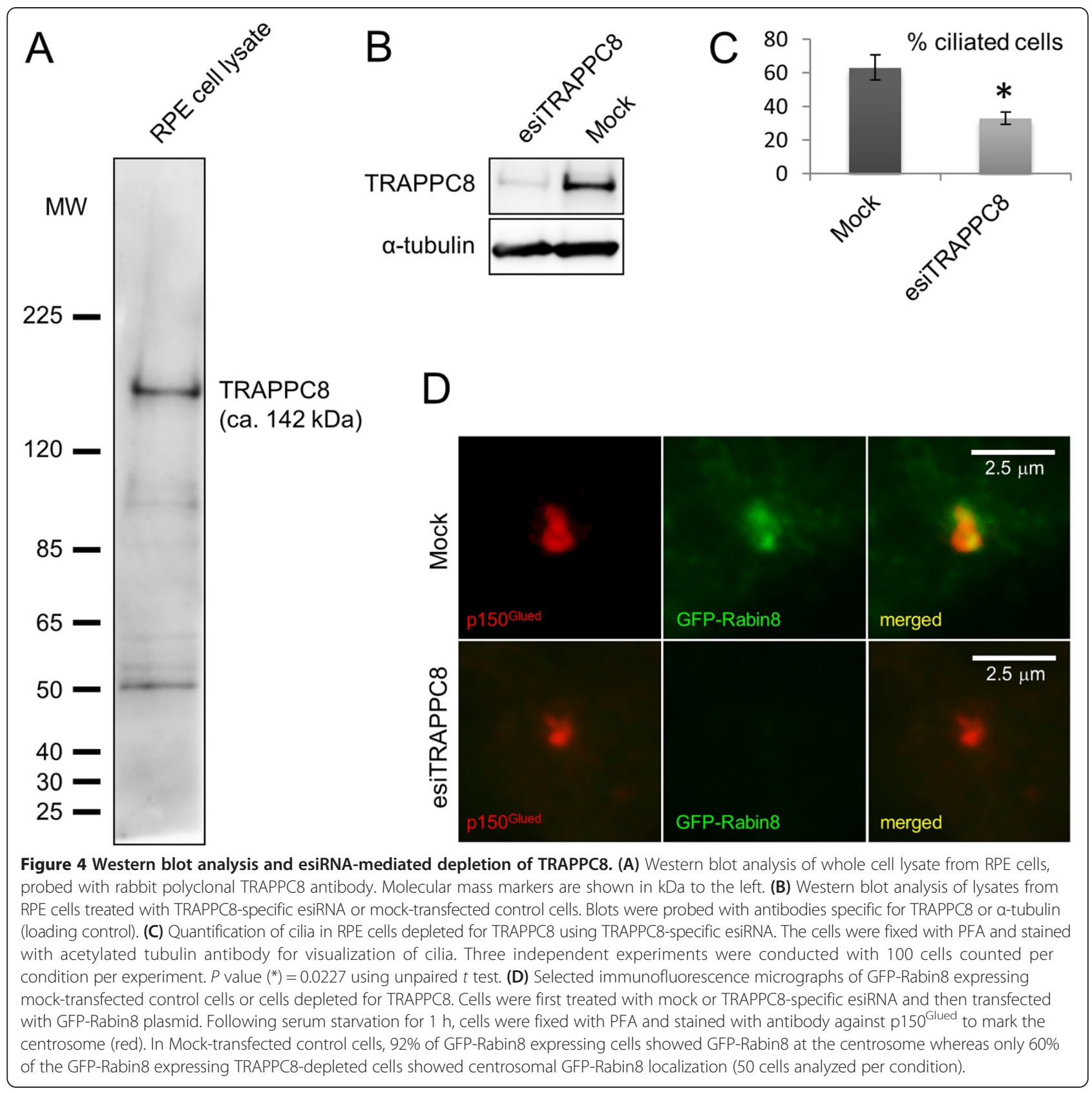

propose that the TRAPPII-specific subunits are paralogous entities that bear conserved domain arrangements consisting of amino terminal arrays of TPR repeats followed by a C-terminal ASH module (except for TRAPPC13, which does not appear to contain an amino terminal TPR repeat region). Among the subunits that adopt such a TPR-ASH bipartite arrangement, we find the yeast TRAPPII Trs120, Trs130, and Trs65 subunits as well as human TRAPPC8, 9, 10, and 11. In addition, we find that these TRAPP components, as with the MSP modules of VAPB [28], show remote homology to the bacterial periplasmic trafficking chaperone PapD of the usher pili assembly machinery [35] (Figures 1 and 2, and Additional file 1: Figure S1). We therefore propose an ancient relationship between the gram-negative bacterial secretory SecY-dependent pili assembly pathway and the eukaryotic TRAPPII-facilitated vesicular trafficking pathway from the late Golgi to the membrane of the primary cilium (Figure 6).

Our immunofluorescence microscopy analysis showing that heterologously expressed TRAPPC10-ASH and TRAPPC11-ASH localize to the centrosome and affect microtubule organization (Figure 3), as well as our observation that endogenous TRAPPC8 localizes to the 


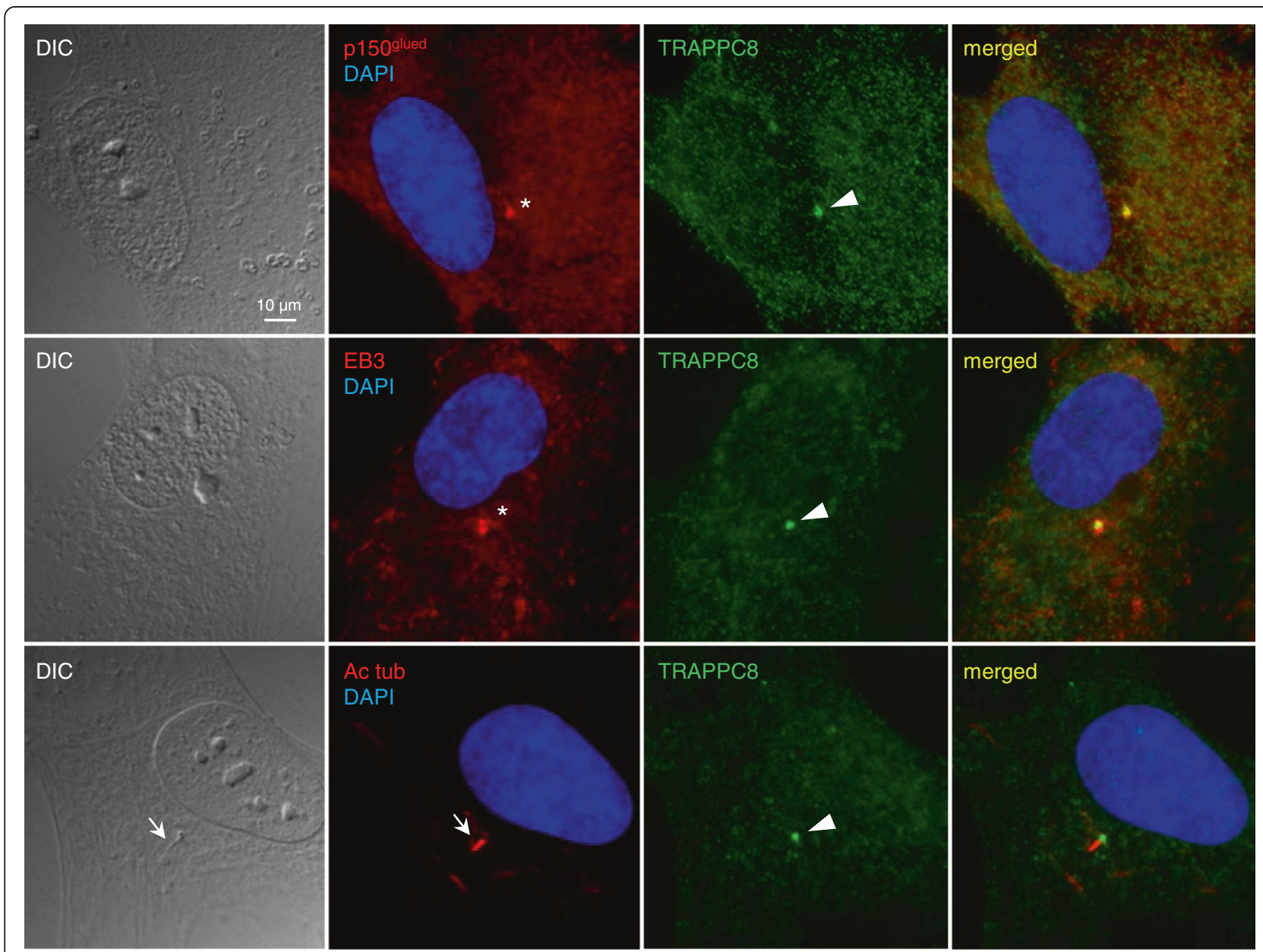

Figure 5 Endogenous TRAPPC8 localizes to the centrosome/basal body. RPE cells were serum-starved for $24 \mathrm{~h}$, fixed with methanol (upper two panels) or PFA (lower panel) and stained with rabbit polyclonal antibody against TRAPPC8 (green) and mouse monoclonal antibody against p150 Glued, rat monoclonal antibody against EB3 or mouse monoclonal antibody against acetylated tubulin (Ac tub), as indicated (red). DNA was stained with DAPI. Arrowheads and asterisks point to the centrosomes/basal bodies. Closed arrow indicates a primary cilium.

centrosome (Figure 5) and is required for ciliogenesis (Figure 4), strongly suggests that the ASH domain constitutes a centrosome-targeting module. How might the ASH domain of TRAPP components confer targeting to the centrosome? One possibility is that the ASH domain directly binds microtubules, since the Drosophila ASPM homolog abnormal spindle, asp, binds microtubules within a 512 amino acid region that contains the ASH domain [50]. Alternatively, it is possible that the TRAPP ASH domain interacts with centrosome-specific Rab GTPases such as Rab8. Structural studies of the ASH domaincontaining OCRL1 protein showed that this domain directly interacts with Rab8a [26], and TRAPPC9 and TRAPPC10 were previously shown to interact with Rabin8 [14], a GTP exchange factor for Rab8 [15]. Finally, a polypeptide comprising the last 211 amino acid residues of TRAPPC9, which includes all of the second ASH domain (Figure 1A), was shown to interact directly with the NIK and IKK $\beta$-binding protein NIB [51], indicating that the
TRAPP ASH domain could also be mediating such interactions. Clearly, more studies are needed to understand how the ASH domain engages in interactions with different binding partners. Such studies will be facilitated by the results of our bioinformatics analysis presented here.

Even though more studies are needed to identify the mechanism by which the ASH domain targets to the centrosome/basal body, several reports indicate that the ASH domains identified here for TRAPPII components are functionally important. For example, a recent report identified a splice site mutation before exon 18 in TRAPPC9, leading to formation of a truncated TRAPPC9 polypeptide ending at amino acid 967, in two patients with a PraderWilli-like phenotype [52], signifying that the C-terminal region of TRAPPC9 containing the second ASH domain (Figure 1A) is functionally important. Similarly, a mutation in TRAPPC11 leading to a single amino acid change in the region between the two predicted $\mathrm{ASH}$ domains (pGly980Arg; Figure 1A) was identified in patients with 


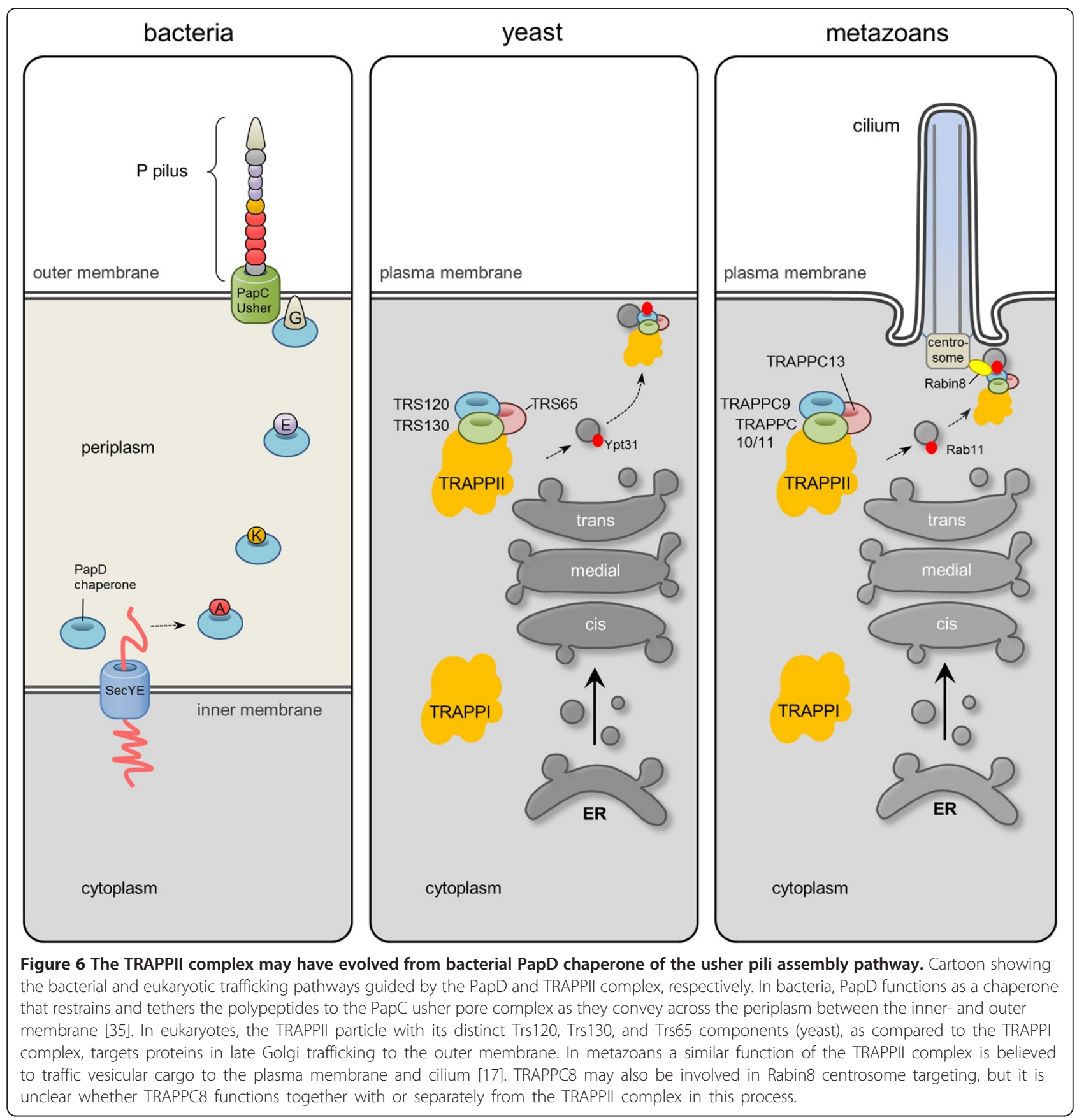

myopathy, infantile hyperkinetic movements, ataxia, and intellectual ability [53], demonstrating the functional importance of this region.

The identification of ASH/MSP domains in the TRAPPII subunits underpins their previously proposed function in ciliary membrane biogenesis [14] at the molecular level, and corroborates the idea that the ASH domain is associated with cilia-related functions [27]. The presence of an amino terminal $\alpha$-helical TPR repeat region is also a hallmark of numerous ciliary proteins [54], and hence the presence of such a TPR repeat region in the N-terminus of most
TRAPPII subunits (Figure 1) is in line with their ciliary function. As with the ASH domain, the TPR repeat region also seems to be functionally important because mutation leading to deletion of residues 372-429 of TRAPPC11 were shown to impair post-Golgi trafficking and to cause myopathy, infantile hyperkinetic movements, ataxia and intellectual disability in patients [53].

Although we found TRAPPC8 to be localized to the centrosome/basal body (Figure 5) and to be required for ciliogenesis and centrosomal targeting of GFP-Rabin 8 (Figure 4) TRAPPC8 has not been reported to be part of 
the TRAPPII complex (see Background). Thus it remains to be determined if the observed effects of TRAPPC8 depletion in ciliogenesis involve interaction with TRAPPII components such as TRAPPC9 and TRAPPC10 [14]. In yeast TRAPPC8 (Trs85) functions in autophagy [17]. Interestingly, two recent studies indicated a functional link between primary cilia and autophagy $[55,56]$, suggesting that TRAPPC8 function at the centrosome/basal body could also be linked to autophagy. In future studies it will be interesting to investigate this further and to investigate possible cilia-related function for TRAPPC11 and TRAPPC13.

\section{Conclusions}

Our results suggest that the TRAPP subunits TRAPPC8, -9, -10 , and -11 subunits as well as their yeast counterparts exhibit a domain structure consisting of an $\mathrm{N}$-terminal $\alpha$-solenoid with TPR repeats followed by a C-terminus harboring one or two ASH domains. Immunofluorescence microscopy analysis as well as esiRNA-mediated silencing of TRAPPC8, indicated that the ASH domain is a bona fide centrosome targeting domain, and that TRAPPC8 has a novel function in promoting ciliogenesis. Human genetics studies suggest that both the TPR repeat region and the ASH domain are functionally important, but more work will be required to investigate the detailed structure and function of these domains. Finally, given the structural similarity between TRAPPCII ASH domains and the bacterial trafficking chaperone PapD of the usher pili assembly machinery, our results indicate that the TRAPPCII components may be evolutionarily related to PapD (Figure 6). This would be in line with the autogenous hypothesis of ciliary evolution in which it is suggested that cilia and IFT evolved from coated vesicle transport $[57,58]$.

\section{Additional file}

Additional file 1: Figure S1. Graphical output showing results of the initial HHpred search with human DLEC1 (residues 769-980) as search query. The amino acid sequence of human DLEC1 (residues 769-980) was used as a search query in HHpred. Profile-to-profile searches were obtained by three iterative PSI-BLAST searches against the PFAM database of HMM profiles. The figure shows a bar graph summarizing the positions and color-coded significances of the database matches with more than $40 \%$ probability. The bars are color-coded according to the significance of the hits (For details see http://toolkit.tuebingen.mpg.de/hhpred). From red meaning very significant to blue meaning not significant.

\footnotetext{
Abbreviations

ASH: ASPM, SPD-2, Hydin; BBS: Bardet Biedl syndrome; BLAST: Basic local alignment search tool; DAPI: 4',6-diamidino-2-phenylindole; DIC: Differential interference contrast; GFP: Green fluorescent protein; HHM: Hidden Markov model; hTERT RPE: Human telomerase-immortalised retinal pigmented epithelial; IFM: Immunofluorescence microscopy; IFT: Intraflagellar transport; MSP: Major sperm protein; MSA: Multiple sequence alignments; OCRL: Oculocerebrorenal syndrome of Lowe protein; PAGE: Polyacrylamide gel electrophoresis; PDB: Protein data bank; PFA: Paraformaldehyde; PSI-BLAST: Position-specific iterative basic local alignment search tool; SDS: Sodium dodecyl sulfate; siRNA: Small interfering RNA; TPR: Tetratricopeptide repeat; TRAPP: Transport protein particle.
}

\section{Competing interests}

The authors declare that they have no competing interests.

\section{Authors' contributions}

KBS carried out the bioinformatics analyses and KBS and SKM performed the experiments. KBS, STC, and LBP made the figures. KBS and LBP wrote the paper. All authors read, edited and approved the final manuscript.

\section{Acknowledgements}

This work was supported by grants from the Lundbeck Foundation, the Novo Nordisk Foundation, the Danish Council for Independent Research, Natural Sciences (grant number 10-085373), the UCPH Excellence Programme for Interdisciplinary Research (2016 Funds), University of Copenhagen, Denmark, and Nordforsk. We thank Dr. Christopher J. Westlake for the kind gift of GFP-Rabin8 plasmid.

\section{Author details}

${ }^{1}$ Department of Biology, University of Copenhagen, Universitetsparken 13 Copenhagen, Denmark. ${ }^{2}$ Center for Experimental Bioinformatics, Department of Biochemistry and Molecular Biology, University of Southern Denmark, Odense, Denmark.

Received: 17 April 2013 Accepted: 22 May 2014

Published: 18 Jun 2014

\section{References}

1. Satir P, Pedersen LB, Christensen ST (2010) The primary cilium at a glance. J Cell Sci 123:499-503

2. Sorokin S (1962) Centrioles and the formation of rudimentary cilia by fibroblasts and smooth muscle cells. J Cell Biol 15:363-377

3. Pedersen LB, Veland IR, Schrøder JM, Christensen ST (2008) Assembly of primary cilia. Dev Dyn 237:1993-2006

4. Ghossoub R, Molla-Herman A, Bastin P, Benmerah A (2011) The ciliary pocket: a once-forgotten membrane domain at the base of cilia. Biol Cell 103:131-144

5. Clement CA, Ajbro KD, de Jesus MPR H, Koefoed K, Vestergaard ML, Veland IR, Pedersen LB, Benmerah A, Andersen CY, Larsen LA, Christensen ST (2013) Regulation of TGF $\beta$ signaling by endocytosis at the pocket region of the primary cilium. Cell Rep 3:1806-1814

6. Hildebrandt F, Benzing T, Katsanis N (2011) Ciliopathies. New Engl J Med 364:1533-1543

7. Waters AM, Beales PL (2011) Ciliopathies: an expanding disease spectrum. Pediatr Nephrol 26:1039-1056

8. Tobin JL, Beales PL (2007) Bardet-Biedl syndrome: beyond the cilium. Pediatr Nephrol 22:926-936

9. Nachury MV, Loktev AV, Zhang Q, Westlake CJ, Peranen J, Merdes A Slusarski DC, Scheller RH, Bazan JF, Sheffield VC, Jackson PK (2007) A core complex of BBS proteins cooperates with the GTPase Rab8 to promote ciliary membrane biogenesis. Cell 129:1201-1213

10. Loktev AV, Zhang Q, Beck JS, Searby CC, Scheetz TE, Bazan JF, Slusarski DC, Sheffield VC, Jackson PK, Nachury MV (2008) A BBSome subunit links ciliogenesis, microtubule stability, and acetylation. Dev Cell 15:854-865

11. Lechtreck KF, Johnson EC, Sakai T, Cochran D, Ballif BA, Rush J, Pazour GJ, Ikebe M, Witman GB (2009) The Chlamydomonas reinhardtii BBSome is an IFT cargo required for export of specific signaling proteins from flagella. J Cell Biol 187:1117-1132

12. Jin H, White SR, Shida T, Schulz S, Aguiar M, Gygi SP, Bazan JF, Nachury MV (2010) The conserved Bardet-Biedl syndrome proteins assemble a coat that traffics membrane proteins to cilia. Cell 141:1208-1219

13. Hsiao YC, Tuz K, Ferland RJ (2012) Trafficking in and to the primary cilium. Cilia 1:4

14. Westlake CJ, Baye LM, Nachury MV, Wright KJ, Ervin KE, Phu L, Chalouni C, Beck JS, Kirkpatrick DS, Slusarski DC, Sheffield VC, Scheller RH, Jackson PK (2011) Primary cilia membrane assembly is initiated by Rab11 and transport protein particle II (TRAPPII) complex-dependent trafficking of Rabin8 to the centrosome. Proc Natl Acad Sci U S A 108:2759-2764

15. Hattula K, Furuhjelm J, Arffman A, Peranen J (2002) A Rab8-specific GDP/ GTP exchange factor is involved in actin remodeling and polarized membrane transport. Mol Biol Cell 13:3268-3280 
16. Knodler A, Feng S, Zhang J, Zhang X, Das A, Peranen J, Guo W (2010) Coordination of Rab8 and Rab11 in primary ciliogenesis. Proc Natl Acad Sci U S A 107:6346-6351

17. Yu S, Liang Y (2012) A trapper keeper for TRAPP, its structures and functions. Cell Mol Life Sci 69:3933-3944

18. Choi C, Davey M, Schluter C, Pandher P, Fang Y, Foster LJ, Conibear E (2011) Organization and assembly of the TRAPPII complex. Traffic 12:715-725

19. Scrivens PJ, Noueihed B, Shahrzad N, Hul S, Brunet S, Sacher M (2011) C4orf41 and TTC-15 are mammalian TRAPP components with a role at an early stage in ER-to-Golgi trafficking. Mol Biol Cell 22:2083-2093

20. Zong M, Wu XG, Chan CW, Choi MY, Chan HC, Tanner JA, Yu S (2011) The adaptor function of TRAPPC2 in mammalian TRAPPs explains TRAPPC2associated SEDT and TRAPPC9-associated congenital intellectual disability. PLOS ONE 6:e23350

21. Schmidt KN, Kuhns S, Neuner A, Hub B, Zentgraf H, Pereira G (2012) Cep164 mediates vesicular docking to the mother centriole during early steps of ciliogenesis. J Cell Biol 199:1083-1101

22. Feng S, Knodler A, Ren J, Zhang J, Zhang X, Hong Y, Huang S, Peranen J, Guo W (2012) A Rab8 guanine nucleotide exchange factor-effector interaction network regulates primary ciliogenesis. J Biol Chem 287:15602-15609

23. Ruellas AC, Pithon MM, Oliveira DD, Oliveira AM (2008) Lowe syndrome: literature review and case report. J Orthod 35:156-160

24. Hyvola N, Diao A, McKenzie E, Skippen A, Cockcroft S, Lowe M (2006) Membrane targeting and activation of the Lowe syndrome protein OCRL1 by rab GTPases. EMBO J 25:3750-3761

25. Fukuda M, Kanno E, Ishibashi K, Itoh T (2008) Large scale screening for novel rab effectors reveals unexpected broad Rab binding specificity. Mol Cell Proteomics 7:1031-1042

26. Hou X, Hagemann N, Schoebel S, Blankenfeldt W, Goody RS, Erdmann KS, Itzen A (2011) A structural basis for Lowe syndrome caused by mutations in the Rab-binding domain of OCRL1. EMBO J 30:1659-1670

27. Ponting CP (2006) A novel domain suggests a ciliary function for ASPM, a brain size determining gene. Bioinformatics 22:1031-1035

28. Tarr DE, Scott AL (2005) MSP domain proteins. Trends Parasitol 21:224-231

29. Bork P, Holm L, Sander C (1994) The immunoglobulin fold. Structural classification, sequence patterns and common core. J Mol Biol 242:309-320

30. Coon BG, Hernandez V, Madhivanan K, Mukherjee D, Hanna CB, BarinagaRementeria Ramirez I, Lowe M, Beales PL, Aguilar RC (2012) The Lowe syndrome protein OCRL1 is involved in primary cilia assembly. Hum Mol Genet 21:1835-1847

31. Lechtreck KF, Witman GB (2007) Chlamydomonas reinhardtii hydin is a central pair protein required for flagellar motility. J Cell Biol 176:473-482

32. Dawe HR, Shaw MK, Farr H, Gull K (2007) The hydrocephalus inducing gene product, Hydin, positions axonemal central pair microtubules. BMC Biol 5:33

33. Lechtreck KF, Delmotte P, Robinson ML, Sanderson MJ, Witman GB (2008) Mutations in Hydin impair ciliary motility in mice. J Cell Biol 180:633-643

34. Mahmood S, Ahmad W, Hassan MJ (2011) Autosomal recessive primary microcephaly (MCPH): clinical manifestations, genetic heterogeneity and mutation continuum. Orphanet J Rare Dis 6:39

35. Waksman G, Hultgren SJ (2009) Structural biology of the chaperone-usher pathway of pilus biogenesis. Nat Rev Microbiol 7:765-774

36. HHpred Homology detection \& structure prediction by HMM-HMM comparison, http://toolkit.tuebingen.mpg.de/hhpred

37. Soding J, Biegert A, Lupas AN (2005) The HHpred interactive server for protein homology detection and structure prediction. Nucleic Acids Res 33:W244-W248

38. MAFFT MAFFT., http://myhits.isb-sib.ch/cgi-bin/mafft

39. Katoh K, Misawa K, Kuma K, Miyata T (2002) MAFFT: a novel method for rapid multiple sequence alignment based on fast Fourier transform. Nucleic Acids Res 30:3059-3066

40. Jalview Jalview., http://www.jalview.org/

41. Modeller Modeller., http://toolkit.tuebingen.mpg.de/modeller

42. Sali A, Potterton L, Yuan F, van Vlijmen H, Karplus M (1995) Evaluation of comparative protein modeling by MODELLER. Proteins 23:318-326

43. Schrøder JM, Larsen J, Komarova Y, Akhmanova A, Thorsteinsson Rl, Grigoriev I, Manguso R, Christensen ST, Pedersen SF, Geimer S, Pedersen LB (2011) EB1 and EB3 promote cilia biogenesis by several centrosome-related mechanisms. J Cell Sci 124:2539-2551

44. Schrøder JM, Schneider L, Christensen ST, Pedersen LB (2007) EB1 is required for primary cilia assembly in fibroblasts. Curr Biol 17:1134-1139
45. Punta M, Coggill PC, Eberhardt RY, Mistry J, Tate J, Boursnell C, Pang N, Forslund K, Ceric G, Clements J, Heger A, Holm L, Sonnhammer EL, Eddy SR, Bateman A, Finn RD (2012) The Pfam protein families database. Nucleic Acids Res 40:D290-D301

46. PDB Protein Data Bank., http://www.pdb.org

47. HNN HNN secondary structure prediction method., http://npsa-pbil.ibcp.fr/ cgi-bin/npsa_automat.pl?page=/NPSA/npsa_hnn.html

48. TPRpred TPRpred., http://toolkit.tuebingen.mpg.de/tprpred

49. Yip CK, Berscheminski J, Walz T (2010) Molecular architecture of the TRAPPII complex and implications for vesicle tethering. Nat Struct Mol Biol 17:1298-1304

50. Saunders RD, Avides MC, Howard T, Gonzalez C, Glover DM (1997) The Drosophila gene abnormal spindle encodes a novel microtubule-associated protein that associates with the polar regions of the mitotic spindle. J Cell Biol 137:881-890

51. Hu WH, Pendergast JS, Mo XM, Brambilla R, Bracchi-Ricard V, Li F, Walters WM, Blits B, He L, Schaal SM, Bethea JR (2005) NIBP, a novel NIK and IKK (beta)-binding protein that enhances NF-(kappa)B activation. J Biol Chem 280:29233-29241

52. Marangi G, Leuzzi V, Manti F, Lattante $S$, Orteschi D, Pecile V, Neri G, Zollino M (2013) TRAPPC9-related autosomal recessive intellectual disability: report of a new mutation and clinical phenotype. Eur J Hum Genet 21:229-232

53. Bogershausen N, Shahrzad N, Chong JX, von Kleist-Retzow JC, Stanga D, Li Y, Bernier FP, Loucks CM, Wirth R, Puffenberger EG, Hegele RA, Schreml J, Lapointe G, Keupp K, Brett CL, Anderson R, Hahn A, Innes AM, Suchowersky O, Mets MB, Nurnberg G, McLeod DR, Thiele H, Waggoner D, Altmuller J, Boycott KM, Schoser B, Nurnberg P, Ober C, Heller R et al (2013) Recessive TRAPPC11 mutations cause a disease spectrum of limb girdle muscular dystrophy and myopathy with movement disorder and intellectual disability. Am J Hum Genet 93:181-190

54. Taschner M, Bhogaraju S, Lorentzen E (2012) Architecture and function of IFT complex proteins in ciliogenesis. Differentiation 83:S12-S22

55. Pampliega O, Orhon I, Patel B, Sridhar S, Diaz-Carretero A, Beau I, Codogno P, Satir BH, Satir P, Cuervo AM (2013) Functional interaction between autophagy and ciliogenesis. Nature 502:194-200

56. Tang Z, Lin MG, Stowe TR, Chen S, Zhu M, Stearns T, Franco B, Zhong Q (2013) Autophagy promotes primary ciliogenesis by removing OFD1 from centriolar satellites. Nature 502:254-257

57. Jekely G, Arendt D (2006) Evolution of intraflagellar transport from coated vesicles and autogenous origin of the eukaryotic cilium. Bioessays 28:191-198

58. Satir P, Mitchell DR, Jekely G (2008) How did the cilium evolve? Curr Top Dev Biol 85:63-82

\subsection{6/2046-2530-3-6}

Cite this article as: Schou et al:: Identification of conserved, centrosometargeting ASH domains in TRAPPII complex subunits and TRAPPC8. Cilia 2014, 3:6

\section{Submit your next manuscript to BioMed Central and take full advantage of:}

- Convenient online submission

- Thorough peer review

- No space constraints or color figure charges

- Immediate publication on acceptance

- Inclusion in PubMed, CAS, Scopus and Google Scholar

- Research which is freely available for redistribution 